\title{
Toward Controlling Coronavirus Disease 2019 (COVID-19) Pandemic by Integrated Registry Systems
}

\author{
Zeinab Mohammadzadeh ${ }^{1}$, Leila Keikha ${ }^{2,{ }^{*}}$ and Elham Maserat ${ }^{1,3}$ \\ ${ }^{1}$ Assistant Professor, Department of Health Information Technology, School of Management and Medical Informatics, Tabriz University of Medical Sciences, Tabriz, Iran \\ ${ }^{2}$ Assistant Professor, Department of Medical Librarianship and Information Sciences, Paramedical School, Zahedan University of Medical Sciences, Zahedan, Iran \\ ${ }^{3}$ Assistant Professor, Department of Medical Informatics, Faculty of Medical Sciences, Tarbiat Modares University, Tehran, Iran \\ "Corresponding author: Department of Medical Librarianship and Information Sciences, Paramedical School, Zahedan University of Medical Sciences, Zahedan, Iran. Email: \\ leilakeikha@gmail.com
}

Received 2020 May 10; Revised 2020 July 12; Accepted 2020 July 24.

Keywords: Coronavirus Disease 2019 (COVID-19), Registry, Data Management

\section{Context}

Several pneumonia cases with unknown etiology first appeared in Wuhan, China, in December 2019. The clinical findings of this disease resembled viral pneumonia, which raised concerns at the Chinese and international levels (1$4)$. The virus spread rapidly throughout the world and affected many people $(1,5)$. The World Health Organization (WHO) named this disease COVID-19. Based on the statistics from the Chinese Center for Disease Control and Prevention, the fatality rate is $2.3 \%$. The number of mortalities is higher in older adults (14.8\% in people over 80 years). Moreover, adults with comorbid diseases (cardiovascular disease, diabetes mellitus, chronic respiratory disease, hypertension, and cancer) are more at risk (6).

Due to the potential and nature of COVID-19, serious surveillance and monitoring systems are needed to assess the status of the disease (7). Disease registration systems can be designed to achieve this goal (8). It is specified as "an organized system that collects the epidemiological and clinical data to evaluate outcomes for a population defined by a particular disease, condition, or exposure" $(9,10))$. Information extracted from these systems is used for evaluating the quality of care and follow-up of patients, creating standards of care, monitoring the disease, community assessment, comparison of processes and care outcomes, evaluating disease control programs, planning, policymaking, improving patient outcomes, research, and education $(11,12)$. All of these goals will be achieved if standard, accurate, and timely data are recorded (13). The experience of similar diseases like SARS (severe acute respiratory syndrome) and MERS (Middle East respiratory syndrome) has confirmed the importance of registry systems in optimal disease management (8).

Developing a registration system for COVID-19 and collecting information about patient history, diagnosis, treatment, and follow-up through electronic systems can lead to better disease management. Moreover, it determines the effectiveness of treatment and clinical interventions for COVID-19 (14). In addition, it offers the opportunity to deepen our understanding of mortality and particularly sudden unexpected death due to COVID-19.

It is important to note that connecting the COVID19 registry system to other registry systems and electronic health records (EHR) can also be used to obtain information about patients or identify people who are at greater risk of this disease. For example, the rheumatology COVID-19 registry collects information about patients with rheumatology who have been affected by COVID-19 (15). CAPACITY-COVID is a European registry to determine the role of cardiovascular diseases in the COVID-19 pandemic. The goal was to manage cardiac complications in patients with COVID-19 and determine the vulnerability of patients with underlying cardiovascular disease to be affected by COVID-19 (16). COVID-19 specific data elements added to the American Heart Association's Get with The Guidelines Registry Modules that is a registry system for capturing data on patients with cardiac disease $(17,18)$. The Sina Hospital COVID-19 registry (SHCo-19R) system collects information about clinical presentations, diagnostic workups, treatments, hospital courses, and follow-ups of patients (19). Isfahan COVID 19 Registry (I CORE) collects data according to the WHO protocols and questionnaires (20). Another study proposed that 44 data items, including clinical and nonclinical data to be collected for COVID19 (8). 
Based on the WHO, the registry of COVID-19 should be composed of four sections, including patient information, clinical information, exposure, and travel information from 14 days before symptom onset, and laboratory information. Demographic data and the place where the case was diagnosed are gathered in the patient information part. Clinical information consists of patient clinical courses, patient symptoms and signs, underlying conditions, and comorbidities. Section 3 collects information on the occupation and travel history from 14 days before symptom onset. Laboratory information is recorded in the final section (21). Detailed information about each section is mentioned below.

\section{Basic information}

This part consists of five data elements, including patient ID, date of birth, sex at the birth, place where the case was first diagnosed, and usual patient place of residency.

\section{Clinical Information}

This section contains four parts, including patient clinical course, patient symptoms, patient signs, and underlying conditions and comorbidities.

\subsection{Patient Clinical Course}

The data elements include the date of symptom onset, admission to the hospital, the date of the first hospital admission, the name of the hospital, the date of isolation, Was the patient ventilated, health status (circle) at the time of reporting, and the date of death are recorded in this part.

\subsection{Patient Symptoms}

They include a history of fever/chills, shortness of breath, pain (check all that apply), general weakness, diarrhea, cough, nausea/vomiting, sore throat, headache, runny nose, irritability/confusion, and so on.

\subsection{Patient Signs}

They include temperature, pharyngeal exudate, coma, abnormal lung X-ray findings, conjunctival injection, dyspnea/tachypnea, seizure, abnormal lung auscultation, and so on.

\subsection{Underlying Conditions and Comorbidity}

Pregnancy (trimester), postpartum ( $<6$ weeks), cardiovascular disease including hypertension, immunodeficiency including HIV, diabetes, renal disease, liver disease, chronic lung disease, chronic neurological or neuromuscular disease, malignancy, and so on are necessary comorbidities that must be recorded.

\section{Occupation and Travel History From 14 Days Before Symptom Onset}

This information includes occupation, places the patient traveled from 14 days before symptom onset, a patient visit to any health care facility from 14 days before symptom onset, close contact with a person with acute respiratory infection from 14 days before symptom onset, contact with a probable or confirmed case from 14 days before symptom onset, and patient visit to any live animal market from 14 days before symptom onset.

\section{Laboratory Information}

This includes the name of confirming laboratory, type of assay and sequencing, and the date of laboratory confirmation (21).

In conclusion, different clinical registry systems have been developed for COVID-19, with different data elements. Due to the importance of registration systems and the variety of clinical registry data items, it is useful to use national and standard data items for COVID-19 clinical registry systems. Although these data are approved by the WHO to record information on suspected or infected patients, they need to be customized for use in every country. For example, in some countries, especially in the basic information section, it may not need to record the ethnicity or race of patients. Different standards need to be considered in designing electronic registration and integration into other systems and EHR. In addition, close collaboration is necessary between clinicians, epidemiologists, health information managers, informatics experts, and even patients to set up a registry system for improving health.

\section{Footnotes}

Authors' Contribution: KL developed the original idea. $\mathrm{KL}$ and MZ drafted the manuscript. KL, MZ, and ME wrote the final manuscript.

Conflict of Interests: The authors declare that there is no conflict of interest.

Funding/Support: None declared by author.

\section{References}

1. Rothan HA, Byrareddy SN. The epidemiology and pathogenesis of coronavirus disease (COVID-19) outbreak. Journal of autoimmunity. 2020:102433. doi: 10.1016/j.jaut.2020.102433. [PubMed:32113704].

2. Singhal T. A review of coronavirus disease-2019 (COVID-19). The Indian Journal of Pediatrics. 2020:1-6. doi: 10.1007/s12098-020-03263-6. [PubMed: 32166607]. 
3. Huang C, Wang Y, Li X, Ren L, Zhao J, Hu Y, et al. Clinical features of patients infected with 2019 novel coronavirus in Wuhan, China. The lancet. 2020;395(10223):497-506. doi:10.1016/S0140-6736(20)30183-5.

4. Murthy S, Gomersall CD, Fowler RA. Care for critically ill patients with COVID-19. Jama. 2020. doi: 10.1001/jama.2020.3633. [PubMed: 32159735].

5. Sun J, He W, Wang L, Lai A, Ji X, Zhai X, et al. COVID-19: epidemiology, evolution, and cross-disciplinary perspectives. Trends in Molecular Medicine. 2020. doi: 10.1016/j.molmed.2020.02.008. [PubMed: 32359479].

6. Alger HM, Williams J, Walchok JG, Bolles M, Fonarow GC, Rutan C. Role of Data Registries in the Time of COVID-19. Circ Cardiovasc Qual Outcomes. 2020:Circoutcomes120006766. doi: 10.1161/circoutcomes.120.006766. [PubMed: 32298145].

7. Sohrabi C, Alsafi Z, O'Neill N, Khan M, Kerwan A, Al-Jabir A, et al. World Health Organization declares global emergency: A review of the 2019 novel coronavirus (COVID-19). International Journal of Surgery. 2020 doi: 10.1016/j.ijsu.2020.02.034. [PubMed: 32112977].

8. Kazemi-Arpanahi H, Moulaei K, Shanbehzadeh M. Design and development of a web-based registry for Coronavirus (COVID-19) disease. Medical Journal of The Islamic Republic of Iran (MJIRI). 2020;34(1):48290.

9. Workman TA. Engaging patients in information sharing and data collection: the role of patient-powered registries and research networks. US: Agency for Healthcare Research and Quality; 2013.

10. Gliklich RE, Dreyer NA, Leavy MB. Registries for evaluating patient outcomes: a user's guide. Government Printing Office; 2014.

11. Bellgard MI, Napier KR, Bittles AH, Szer J, Fletcher S, Zeps N, et al. Design of a framework for the deployment of collaborative independent rare disease-centric registries: Gaucher disease registry model. Blood Cells, Molecules, and Diseases. 2018;68:232-8. doi: 10.1016/j.bcmd.2017.01.013. [PubMed: 28190666].

12. Mohammadzadeh Z, Ghazisaeedi M, Nahvijou A, Kalhori SRN, Davoodi S, Zendehdel K. Systematic review of hospital based cancer registries (HBCRs): necessary tool to improve quality of care in cancer patients. Asian Pacific journal of cancer prevention: APJCP. 2017;18(8):2027

13. Hashemi-Shahri SM, Khammarnia M, Ansari-Moghaddam A, Setoodehzadeh F, Okati-Aliabad H, Peyvand M. Sources of news as a necessity for improving community health literacy about COVID-19. Medical Journal of The Islamic Republic of Iran (MJIRI). 2020;34(1):453-5.

14. Qiu R, Wei X, Zhao M, Zhong C, Zhao C, Hu J, et al. Outcome reporting from protocols of clinical trials of Coronavirus Disease 2019 (COVID19): a review. medRxiv. 2020. doi:10.1101/2020.03.04.20031401.

15. Robinson PC, Yazdany J. The COVID-19 Global Rheumatology Alliance: collecting data in a pandemic. Nature Reviews Rheumatology. 2020:1-2. doi: 10.1038/s41584-020-0418-0. [PubMed: 32242121].

16. Asselbergs FW. CAPACITY-COVID: a European registry to determine the role of cardiovascular disease in the COVID-19 pandemic. European Heart Journal. 2020.

17. Alger HM, Williams IV JH, Walchok JG, Bolles M, Fonarow GC, Rutan C. Role of Data Registries in the Time of COVID-19. Circulation: Cardiovascular Quality and Outcomes. 2020;13(5). e006766. doi: 10.1161/CIRCOUTCOMES.120.006766. [PubMed: 32298145].

18. Raeisi A, Tabrizi JS, Gouya MM. IR of Iran National Mobilization against COVID-19 Epidemic. Archives of Iranian medicine. 2020;23(4):216. doi: 10.34172/aim.2020.01. [PubMed: 32271593].

19. Talebpour M, Hadadi A, Oraii A, Ashraf H. Rationale and Design of a Registry in a Referral and Educational Medical Center in Tehran, Iran: Sina Hospital Covid-19 Registry (SHCo-19R). Advanced Journal of Emergency Medicine. 2020;4(2s):e53.

20. Javanmard SH, Nasirian M, Ataei B, Vaseghi G, Vaezi A, Changiz T. Isfahan COvid-19 REgistry (I-CORE): Design and methodology. Journal of Research in Medical Sciences. 2020;25(1):32. doi: 10.4103/jrms.JRMS_271_20. [PubMed: 32582338].

21. World Health Organization. Revised case report form for confirmed Novel Coronavirus COVID-19. World Health Organization; 2020. Available from: https://apps.who.int/iris/handle/10665/331234. 